\title{
In-Silico Screening of Flavonoids Targeted for Death Receptors in Cancer by Using Hex Molecular Docking
}

\author{
Vaithiyalingam Jagannathan Vishnuvarthan*, Karunanidhi Santhanam Lakshmi, Ammayappan Rajam Srividya \\ Department of Pharmacology, SRM College of Pharmacy, SRM University, Kattankulathur, Tamil Nadu, INDIA.
}

\begin{abstract}
Objective: Docking is one of the major tools in the drug development process, here we have selected certain flavonoid molecules such as Formononetin, Tangeritin, Myricetin and Kaempferol and we docked against Death Receptors (DRs) for the prevention of cancer progression. Materials and Methods: In this study, Protein Ligand Docking, we have used HEX as a Docking Software. Receptor structure was obtained from Protein Data Bank (PDB) while the ligand is drawn by using the Chem Draw Software and docking was done according to the specified parameters. Results: All the investigatory molecules except tangeritin (DR5 -212.2; DR 2 -231.1) showed higher energy values on the Death Receptor 5 (Formononetin DR5 -197.8) which states, these flavonoid molecules are having higher affinity and steric compatibility to Death Receptor 5, where DR5 mediates the TRAIL mediated apoptosis. Tangeritin molecule shows higher energy values at DR2 -231.1, it may mediate its effect through the Fas apoptotic path. The Docked molecules are viewed with the help of chimera software to discover the interaction of molecules with the receptors. Conclusion
\end{abstract}

From the present study, it is confirmed that these investigatory molecules showed a predictable effect over Death Receptors, and further studies will be carried out with ADME/T (Absorption, Distribution, Metabolism, Elimination, Toxicity) Tool.

Key words: Flavonoids, Docking, Hex, Death Receptors.

Correspondence :

Mr.V.J.Vishnu Varthan M.Pharm., (Ph.D), Research Scholar, SRM College of Pharmacy, SRM University, Kattankulathur, Chengalpattu District, Kattankulathur, Tamill Nadu, INDIA.

Phone no: 09894699234

Email: vishnuvj24@gmail.com

DOI: 10.5530/jyp.2017.9.33

\section{INTRODUCTION}

Cancer is an unregulated uncontrolled growth of cells which occurs in any part of the body, which is one of the leading causes of death worldwide. According to International Agency for Research on cancer, it was projected about $6 \%$ of death in India is due to cancer. ${ }^{1}$ Also World Health Organization (WHO) has proposed around 7,00,000 people were affected by cancer in India, which may be due to the increase in population, urbanization, industrialization and lifestyle changes.

Various treatment process for cancer like surgery, radiation and chemotherapy still exist, but has some drawbacks in eradicating cancer from human society, which may be due to lack of cell cycle specificity, toxicity of chemotherapy drugs and development of drug resistance. This made the researchers to work on the development of new molecules in cancer treatment. ${ }^{2}$

One of the targeted sites for the cancer treatment is activation of apoptosis either extrinsic or intrinsic pathway. Apoptosis is a highly programmed cell death for tissue remodeling, homeostasis and development of multicellular organisms. ${ }^{3}$ Extrinsic pathway of apoptosis is associated with death receptor stimulation on the cell surface, these death receptors belong to the tumor necrosis factor receptor (TNFR) superfamily with TNF-R1, CD95 (APO-1, Fas), TRAMP (APO-3 DR-3), TNF-related apoptosis-inducing ligand (TRAIL)- Receptor 1 (TRAIL-R1 DR4) and TRAIL - R2 (APO-2, DR5). It is better to target the molecule on the death receptors (site specific) for producing better therapeutic action of the lead molecules and also helps in reducing the side effects of molecules at the other regions. Among the death receptors DR3 doesn't have enough detail mechanism in activation of apoptosis, ${ }^{4}$ also evidences that DR3 shows activation of apoptosis in low, dense population of osteoblasts, while in high density population it doesn't show apoptosis. ${ }^{5}$
The receptor structures are obtained from Protein Data Bank (PDB) and the receptors are selected based on the previous studies. The Death Receptor 1 (1F3V), ${ }^{6}$ Death Receptor 2 (3MX7), ${ }^{7}$ Death Receptor 4 (1DG6), ${ }^{8}$ Death Receptor 5 (1D4V). ${ }^{9}$

Many compounds which are in use today are the derivatives of natural products. Among the most widely used drugs which derived from plant alkaloids are the taxanes, Vinca alkaloids and topoisomerase inhibitors. Antitumor agents such as antibiotic, anthracyclines, Bleomycin and Mitomycin-C. Several antimetabolites also origins of natural products. ${ }^{10}$ Flavonoids act as promising agents in combination with several chemotherapeutic agents against the growth of tumor cells. ${ }^{11}$ Some mechanism involved in those activities are through cell cycle modulation, antiangiogenesis, apoptosis induction, anti-oxidant, prevention of carcinogenic metabolic activation. Flavonoids in combination with chemotherapy to help overcome the problem of resistance of cancer cells and increases the effectiveness of chemotherapeutic agents.

There are various methods available for the drug development like combinatorial chemistry, High Throughput Screening, Denovo synthesis, Computational methods, etc. Bioinformatics is an interdisciplinary field which is comprised of Science, Computer Science, Statistics, Mathematics and engineering, helps in development of methods and software tools for understanding biological data. Bioinformatics has been used for In Silico analyses of biological queries using mathematical and statistical techniques. ${ }^{12}$

Computational Biology and Bioinformatics contributes their role, not only speeding up the drug discovery process,also helps in reducing the costs invested in designing of drugs. Rational Drug Design (RDD) helps 
to facilitate and speed up the drug designing process, which involves a variety of methods to identify novel compounds. ${ }^{8}$ One such method is the docking of the drug molecule with the receptor (target). The site of drug action, which is ultimately responsible for the pharmaceutical effect, is a receptor. Docking is the process by which two molecules fit together in 3D space. Here we have used Hex as Docking Software version 8.0.0 and the interaction of the ligand-protein binding was studied with software chimera version 1.10.2.

The purpose of the study was to identify the potential phytochemicals, which binds to the death receptors for the activation of apoptosis in cancer cells. The data collected here shows the possibility of flavonoids, which successfully binds to the death receptors for initiation of cell death in cancer cells. This data may break through the lead molecules for further investigation in cancer studies.

\section{MATERIAL AND METHODS}

Bioinformatics tools such as PubMed Central (PMC), Drug Bank, PDB (Protein Data Bank) and the software's like Hex, Chem draw was used in the present study. Chem draw is a professional tool for researchers to design and communicate the chemical structures also it helps to draw the chemical molecules and calculates chemical properties.

Hex is an interactive molecular graphics tool for calculating and displaying feasible docking modes of pairs of protein and DNA molecules. Hex also calculates protein-ligand Docking, assuming ligand is rigid and it can superimpose molecules in $3 \mathrm{D}$ shapes. It uses spherical polar Fourier correlations to accelerate the calculations and it's still one of the few docking programs which has built-in graphics to view the results. ${ }^{13}$

Drug bank is an online database containing information regarding drug and drug targets. Drug Bank provides complete information of chemical, pharmacological, pharmaceutical, sequencing of a drug target, structure and pathway of molecular interactions. It is widely used by the drug industry, Pharmacist and physicians. ${ }^{14}$

Three dimensional structural database of the biological molecules such as nucleic acids, proteins was provided by the Protein Data Bank (PDB). $\mathrm{PDB}$ is online free accessible key resource in structural biology and
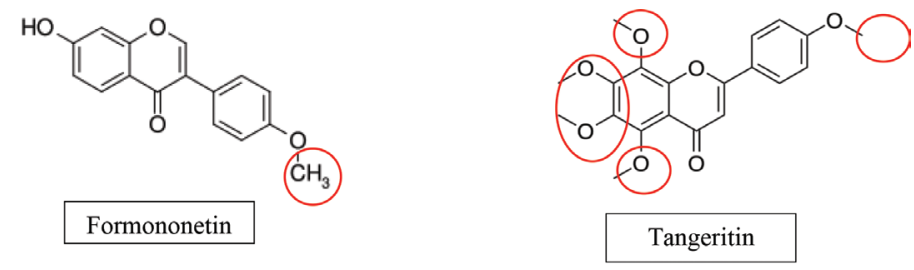<smiles>O=c1c(O)c(-c2cc(O)c(O)c(O)c2)oc2cc(O)cc(O)c12</smiles><smiles>O=c1c(O)c(-c2ccc(O)cc2)oc2cc(O)cc(O)c12</smiles>

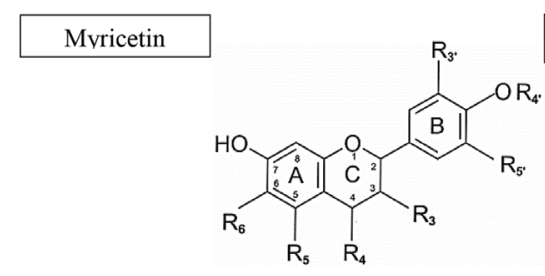

Kaempferol

General Structure of Flavonoids

Figure 1: Structure of flavonoids.

structural genomics, which provides information, regarding the protein obtained by X-ray crystallography, NMR spectroscopy and Cyro - electron microscopy. ${ }^{15}$

Among the digital media, which publishes scholarly articles in the field of biomedical and life science journal, PubMed Central (PMC) plays a significant role in providing information of journals, scientific research data's which is a major research database in suite of resources developed by the National center for Biotechnology Information (NCBI).

The structure of Death Receptors (DR) was obtained from the PDB, It is shown in the Figure 2, while the structure of flavonoids was sketched using Chem draw and the docking analysis of flavonoids with Death Receptors was carried by using the HEX docking Software.

The parameters used in the docking process were

$\begin{array}{lll}\text { - } & \text { Correlation type } & - \\ \text { - } & \text { FTT mode } & \text { - 3D Fast lite } \\ \text { - } & \text { Grid Dimension } & -0.6 \\ \text { - } & \text { Receptor Range } & -180 \\ \text { - } & \text { Ligand Range } & -180 \\ \text { - } & \text { Twist Range } & -360 \\ \text { - } & \text { Distance Range } & -40\end{array}$

The flavonoids are docked using the above conditions with the death receptors.

\section{RESULTS AND DISCUSSION}

Docking results are tabulated in Table 1, between the flavonoids and Death Receptors Hex software allows the receptor molecule to rotate on the $\mathrm{Z}$ axis, ${ }^{16}$ from the obtained results Tangeritin molecule has a higher energy value of all the Death receptors than compared to any other molecule. Its shown in Table 1.

The circled parts depicted in Figure 1 distinguish the difference between the flavonoid molecules. The receptor family was selected from the Protein Data Bank (PDB). Death Receptor 3 was not selected for the present study because of the DR3

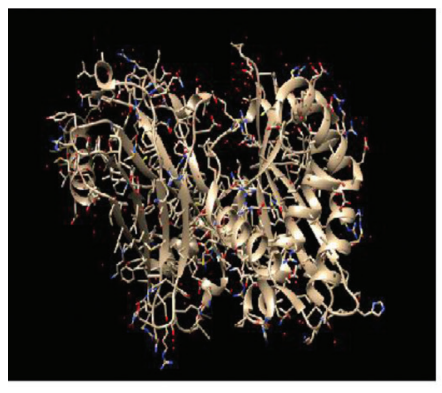

Death Receptor 1

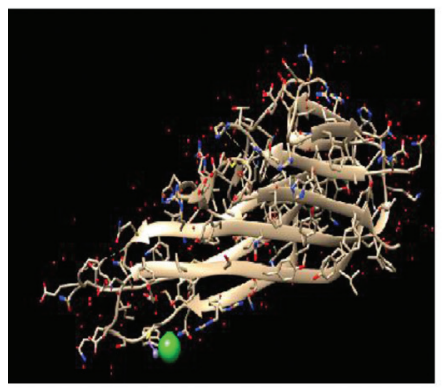

Death Receptor 4

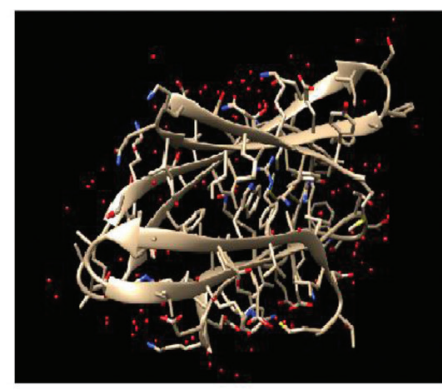

Death Receptor 2

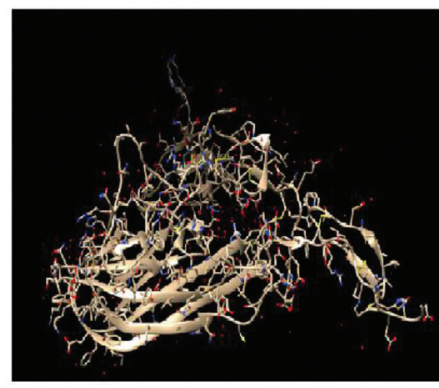

Death Receptor 5
Figure 2: Structure of Death Receptors. 
Table 1: Effect of Investigatory molecules over the Receptor

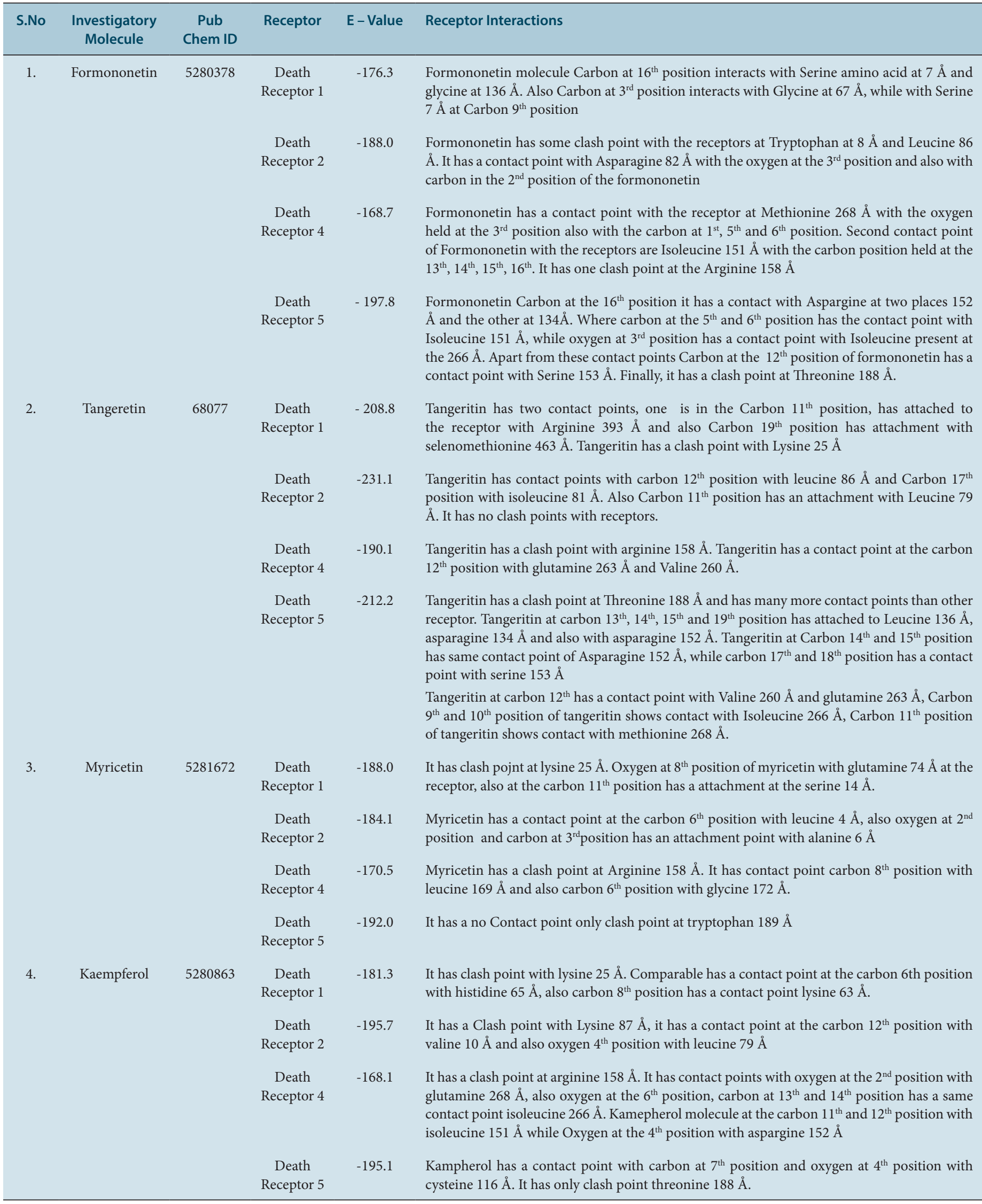


do not relate in regulating the cell death. DR 3 plays a major role in the development of experimental autoimmune encephalomyelitis, allergic lung inflammation, inflammatory arthritis. ${ }^{17}$

From the results, all the investigatory molecules except tangeritin (DR5 -212.2; DR 2 -231.1) showed higher energy values on the Death Receptor 5 (Formononetin DR5 -197.8) which states, these flavonoid molecules are having higher affinity and steric compatibility to Death Receptor 5, where DR5 mediates the TRAIL mediated apoptosis. Tangeritin molecule shows higher energy values at DR2 -231.1, it may mediate its effect through the Fas apoptotic path. The Docked molecules are viewed with the help of chimera software to discover the interaction of molecules with the receptors.

\section{CONCLUSION}

For the Structural based drug designing, protein-ligand interaction plays a significant role, so in the present work we have selected the receptors and the investigatory molecule was used against the Death Receptors. When molecules docked with death receptors except Tangeritin all other molecules showed higher energy state at Death Receptor 5 while Tangeritin showed higher energy value at Death Receptor 2. Further, these works may be extended for ADME/T (Absorption, Distribution, Metabolism, Excretion / Toxicity) of these compounds using commercially available ADME/T tools which helps in the Drug Discovery and Drug development process.

\section{ACKNOWLEDGEMENT}

Authors would like to thank SRM University for providing this opportunity to carry out this study.

\section{CONFLICT OF INTEREST}

The Authors declare that there is no conflict of interest.

\section{ABBREVIATION USED}

DR: Death Receptor; NCBI: National Centre for Biotechnology Information; ADME: Absorption, Distribution, Metabolism, Excretion.

\section{REFERENCES}

1. Rajesh D. Cancer mortality in India: a nationally representative survey'. Lancet. 2012;379(1):1807-17

2. Caroline Belchamber. The diagnosis and treatment of lung cancer (update). National Collaborating Center of Cancer. 2013;22:1-198.

3. Henning W, Kerstin P, Stefanie M. Cordier. Death Receptors as targets for anticancer therapy. J Mol Med. 2008;12(6B):2566-85.

4. Wang Y. On Death Receptors 3 and its Ligands. J Cell Molecule System Tech. 2012;137:114-16.

5. Borysenko CW, Garcia-Palacios V, Griswold RD, Li Y, Iyer AK, Yaroslavskiy BB et al. Death Receptor -3 mediates apoptosis in human osteoblasts under narrowly regulated conditions. J Cell Physio. 2006; 209(3):1021-8.

6. Yukata O, Yuri M, Takehiro S. Highly Precise Protein-Protein Interaction Prediction Based on Consensus Between Template Based and de novo Docking Methods', BMC Proc. 2013;7(7):1-10

7. Changwen J, Guoming L, Linglong O, Shuaipeng M, Yujie W. Structure Determination of Human Fas Apoptosis Inhibitory Molecule and Identification of the Critical Residues Linking the Interdomain Interactions to the Anti-Apoptotic Activity. Acta Cryst. 2014;70:1812-22.

8. Lukasz k, Marcin M, Tuo C, Bin X, Yaogi Z, Keith D. In-Silico Prediction of Disorder Content Using Hybrid Sequence Representation. BioMed Central Bioinforma. 2011;12(1):1-16

9. Koki T, Tatsuya Y, Kiyonobu Y, Yuichiro H. The Development of an Affinity Evaluation and Prediction System by Using Protein-Protein Docking Simulations and Parameters Tuning', Adv Appl in Bioinforma chem. 2009;2:1-15.

10. Edy M, Adam H, Anindyajati. Natural Products for Cancer-targeted therapy: Citrus flavonoids as Potent Chemopreventive agents. Asian Pac J cancer Prev. 2012;13(1):427-37.

11. Peter $M$, Tarek M. Current and emerging medical treatments for non-small cell lung cancer: A primer for pulmonologists. Respiratory Med. 2012;106(1):473-92.

12. Hageway P, Searls David B. The roots of Bioinformatics in theoretical biology. Computational Biology. 2011;7(3):73-5

13. Halperin, Wolfson. Principles of Docking: an overview of search algorithms and a guide to scoring functions. Proteins. 2002;47(4):409-43

14. Ritchie DW, Kemp GJL. Protein Docking using spherical polar Fourier correlation Proteins: Structural and functional Genetics. 2000;39(2):178-94.

15. Wishart DS, Knox C, Guo AC. DrugBank: a comprehensive resource for in silico drug discovery and exploration. Nucleic Acids Res. 2006;34(1):668-72.

16. Berman, HM. The Protein Data Bank: a historical perspective. Acta Crysta Section A: Foundations of Crystallography. 2008;A64(1):88-95

17. Kazemi BA, Karmi SM, kabiri HR, Tavakoli K. Docking Studies of Competitive Interaction of Human Serum Albumin with Ibuprofen and aspirin using Hex Docking Software. J Emerging Trends in Computing and Information Sci. 2013;4(1):97-9. 